\title{
Mid-Regional Proadrenomedullin and Mid- Regional Proatrial Natriuretic Peptide Clearance Predicts Poor Outcomes Better Than Single Baseline Measurements in Critically Ill Patients With Pneumonia: A Retrospective Cohort Study
}

Jos Van Oers ${ }^{1}$, Johannes Krabbe ${ }^{2}$, Evelien Kemna ${ }^{2}$, Yvette Kluiters ${ }^{3}$, Piet Vos ${ }^{1}$, Dylan De Lange ${ }^{4}$, Armand Girbes ${ }^{5}$, Albertus Beishuizen ${ }^{6}$

1. Department of Intensive Care Medicine, Elisabeth-TweeSteden Ziekenhuis, Tilburg, NLD 2. Department of Clinical Chemistry, Medisch Spectrum Twente, Enschede, NLD 3. Department of Clinical Chemistry, Elisabeth-TweeSteden Ziekenhuis, Tilburg, NLD 4. Department of Intensive Care Medicine, University Medical Centre Utrecht, University Utrecht, Utrecht, NLD 5. Department of Intensive Care Medicine, Amsterdam University Medical Center, Medical Centres, VU University Medical Centre, Amsterdam, NLD 6. Department of Intensive Care Medicine, Medisch Spectrum Twente, Enschede, NLD

Corresponding author: Jos Van Oers, jvanoers15@gmail.com

\section{Abstract}

\section{Background}

We assessed the ability of baseline and serial measurements of mid-regional proadrenomedullin (MRproADM) and mid-regional proatrial natriuretic peptide (MR-proANP) to predict 28-day mortality in critically ill patients with pneumonia compared with Acute Physiological and Chronic Health Evaluation IV (APACHE IV) model and Sequential Organ Failure Assessment (SOFA) score.

\section{Methodology}

Biomarkers were collected for the first five days in this retrospective observational cohort study. Biomarker clearance (as a percentage) was presented as biomarker decline in five days. We investigated the relationship between biomarkers and mortality in a multivariable Cox regression model. APACHE IV and SOFA were calculated after 24 hours from intensive care unit admission.

\section{Results}

In 153 critically ill patients with pneumonia, 28-day mortality was $26.8 \%$. Values of baseline MR-proADM, MR-proANP, and APACHE IV were significantly higher in 28-day nonsurvivors, but not significantly different for SOFA score. Baseline MR-proADM and MR-proANP, APACHE IV, and SOFA had a low area under the curve in receiver operating characteristics (ROC) curves. No optimal cut-off points could be calculated. Biomarkers and severity scores were divided into tertiles. The highest tertiles baseline MR-proADM and MRproANP were not significant predictors for 28-day mortality in a multivariable model with age and APACHE IV. SOFA was not a significant predictor in univariable analysis. Clearances of MR-proADM and MR-proANP were significantly higher in 28-day survivors. MR-proADM and MR-proANP clearances had similar low accuracy to identify nonsurvivors in ROC curves and were divided into tertiles. Low clearances of MRproADM and MR-proANP (first tertiles) were significant predictors for 28-day mortality (hazard ratio [HR]: 2.38; 95\% confidence interval [CI]: $1.21-4.70 ; \mathrm{p}=0.013$ and HR: $2.27 ; 95 \% \mathrm{CI}: 1.16-4.46 ; \mathrm{p}=0.017$ ) in a model with age and APACHE IV.

\section{Conclusions}

MR-proADM and MR-proANP clearance performed better in predicting 28-day mortality in a model with age and APACHE IV compared with single baseline measurements in a mixed population of critically ill with pneumonia.

Categories: Anesthesiology, Infectious Disease, Pulmonology

Keywords: biomarkers, pneumonia, mr-proadm, mr-proanp, apache iv, sofa

\section{Introduction}

Pneumonia is an important reason for intensive care unit (ICU) admission, length of stay (LOS), and death [1-3]. Mortality rates for community-acquired pneumonia (CAP) admitted to the ICU of $20-30 \%$ are reported [4]. For hospital-acquired pneumonia (HAP), mortality rates may be as high as 30 to $70 \%$ [5], and attributable mortality of 33 to $50 \%$ in patients with ventilator-associated pneumonia (VAP) has been reported [5]. 
Knowledge of prognostic factors predicting outcome in pneumonia may help grade its severity and predict treatment response.

The Acute Physiological and Chronic Health Evaluation IV (APACHE IV) model and the Sequential Organ Failure Assessment (SOFA) score were developed to assess disease severity or severity of organ dysfunction and predict outcome in critically ill patients [6]; however, their complexity hampers their incorporation in daily routine [6]. Additionally, biomarkers have been proposed as surrogates for these clinical scores to predict outcomes. Conventional biomarkers such as white blood count (WBC), C-reactive protein (CRP), procalcitonin (PCT), and lactate have low prognostic value in predicting mortality in patients with CAP or sepsis [7-9]. There may be a role for new cardiovascular biomarkers, namely, mid-regional proadrenomedullin (MR-proADM) and mid-regional proartrial natriuretic peptide (MR-proANP), to predict mortality in critically ill patients with pneumonia. We investigated the role of the precursor fragments of the prohormones of adrenomedullin (ADM) and atrial natriuretic peptide (ANP) because they are more stable than their respective hormones [10,11], making these assays more feasible for clinical purposes. MRproADM is the mid-region part of the prohormone of $A D M$, a peptide released by multiple tissues with the anti-inflammatory and antiapoptotic effects on vascular endothelial cells, protecting the microcirculation against endothelial permeability in sepsis [12]. Moreover, ADM enhances cardiac output [9]. MR-proADM levels are rapidly induced in lower respiratory tract infections $[7,9,13]$. Baseline MR-proADM measurements are proven to be a good predictor of both short and long-term survival in CAP patients admitted to the emergency room or ICU $[7,9]$. Clearance in serial MR-proADM levels of $30 \%$ or more within five days in septic patients admitted to the ICU was associated with better outcomes [14]. MR-proANP is the mid-region part of the prohormone of ANP, a hormone predominantly produced in the atrium of the heart. ANP antagonizes the renin-angiotensin-aldosterone system in response to hypertension and water and salt retention [15]. Increased levels of MR-proANP have been reported in nonsurvivors of patients with CAP, VAP, and sepsis [15-17].

In the present study, we aimed to investigate the prognostic value of MR-proADM and MR-proANP at baseline compared with the APACHE IV model and SOFA score to predict 28-day mortality in a cohort of critically ill patients with pneumonia of any cause. Our secondary aim was to predict 28-day mortality by clearance of MR-proADM and MR-proANP using serial measurements during five days in comparison with the APACHE IV model and SOFA score. Preliminary results of this study were previously presented as a meeting abstract at the Society of Critical Care Medicine Annual Scientific Meeting on February 26, 2018.

\section{Materials And Methods Study design and selection criteria}

This observational cohort study is a post hoc analysis of the Stop Antibiotics on Procalcitonin Guidance Study (SAPS) [18], a randomized controlled trial, in which the efficacy and safety of PCT guidance in reducing the duration of antibiotic treatment in critically ill patients in 15 Dutch ICUs were investigated from 2009 until 2013. Clinical data and microbiological and laboratory results were prospectively collected during this period. The study protocol was approved by the ethics committee of the VU University Medical Centre (Amsterdam, Netherlands). Informed consent was obtained from all participating patients of SAPS, and all patients agreed that blood samples were stored for further research. Eligible patients were critically ill patients with pneumonia from one of the participating centers of the SAPS trial (Elisabeth-TweeSteden hospital). MR-proADM and MR-proANP were measured in samples of these patients. Exclusion criteria include age $<18$ years, no diagnosis of pneumonia, incomplete data to calculate APACHE IV and SOFA at baseline, and unavailability of plasma samples on admission. Pneumonia was characterized by a new or progressive and persistent infiltrate on chest imaging together with fever, leukocytosis, leukopenia, or altered mental status, and at least one of the following: new onset of purulent sputum, new-onset cough, rales or bronchial breath sound, or worsening gas exchange [19]. CAP is defined as pneumonia acquired outside a hospital or a long-term care facility [1-3]. HAP refers to pneumonia that occurs 48 hours or more after admission [5]. VAP is defined as pneumonia that arises more than 48-72 hours after endotracheal intubation [5]. We followed the Strengthening the Reporting of Observational Studies in Epidemiology Statement guidelines for reporting observational studies [20].

\section{Procedures}

Blood samples were collected in EDTA tubes on a daily basis during the SAPS trial. Plasma was separated by centrifugation and stored in aliquots at $-80^{\circ} \mathrm{C}$. MR-proADM and MR-proANP concentrations were retrospectively measured using an automated immunofluorescent sandwich assay on a Kryptor Compact Plus analyzer (Brahms AG, Henningsdorf, Germany) at the clinical laboratory in Enschede, Netherlands. The Kryptor measures the signal emitted from an immunocomplex by time-resolved amplified cryptate emission. MR-proADM and MR-proANP assay have a limit of detection of $0.05 \mathrm{nmol} / \mathrm{L}$ and $2.1 \mathrm{pmol} / \mathrm{L}$ and functional sensitivity (lowest value with an interassay coefficient of variation $[\mathrm{CV}]<20 \%$, as described by the manufacturer) of $0.23 \mathrm{nmol} / \mathrm{L}$ (MR-proADM) and $4.5 \mathrm{pmol} / \mathrm{L}$ (MR-proANP), respectively. The imprecision of both assays was verified according to the Clinical \& Laboratory Standards Institute Evaluation Protocol 15 (CLSI EP15), using a low and high sample, measured for five days in triplicate. Between- and within-run CVs were all below 5\%. APACHE IV and SOFA were extracted from the SAPS database. 


\section{Statistical analysis}

All non-normally distributed data (Kolmogorov-Smirnov test; $\mathrm{p}<0.05$ ) were expressed as median (with interquartile range, IQR) or as the number of patients (percentage) where appropriate. Patient characteristics and outcomes were compared using a Mann-Whitney U-test for skewed distributed continuous variables and a chi-square test for categorical variables. Clearances of MR-proADM and MR-proANP were calculated by dividing the decline in biomarkers from day one to day five by the value of day one and were presented as percentages. The association between mortality and each biomarker and clinical score at admission and clearances of biomarkers was assessed using the area under the receiver operating characteristics (ROC) curves. Biomarkers and clinical scores at admission and biomarker clearance were separated in tertiles. Univariable and multivariable Cox proportional hazards regression analyses were done to study the effects on the outcome. Potential confounding variables were selected based on univariable regression analysis (variables that yielded $\mathrm{p}<0.05$ ) and were subsequently included in the multivariable regression analysis. The model was checked for intercorrelations among the predictor variables by collinearity statistics. All tests were two-sided and a p-value of $<0.05$ was considered statistically significant. All data were analyzed using the Statistical Package for the Social Sciences version 24 (IBM Corp., Armonk, NY, USA).

\section{Results}

\section{Descriptive characteristics of the patients}

We selected a cohort of 210 patients with pneumonia from the original SAPS database. In 153 patients, MRproADM and MR-proANP concentrations were measured at baseline in addition to APACHE IV and SOFA scores. The patient flow diagram shows the flow of patients along with the primary endpoint of 28-day survival (Figure 1).

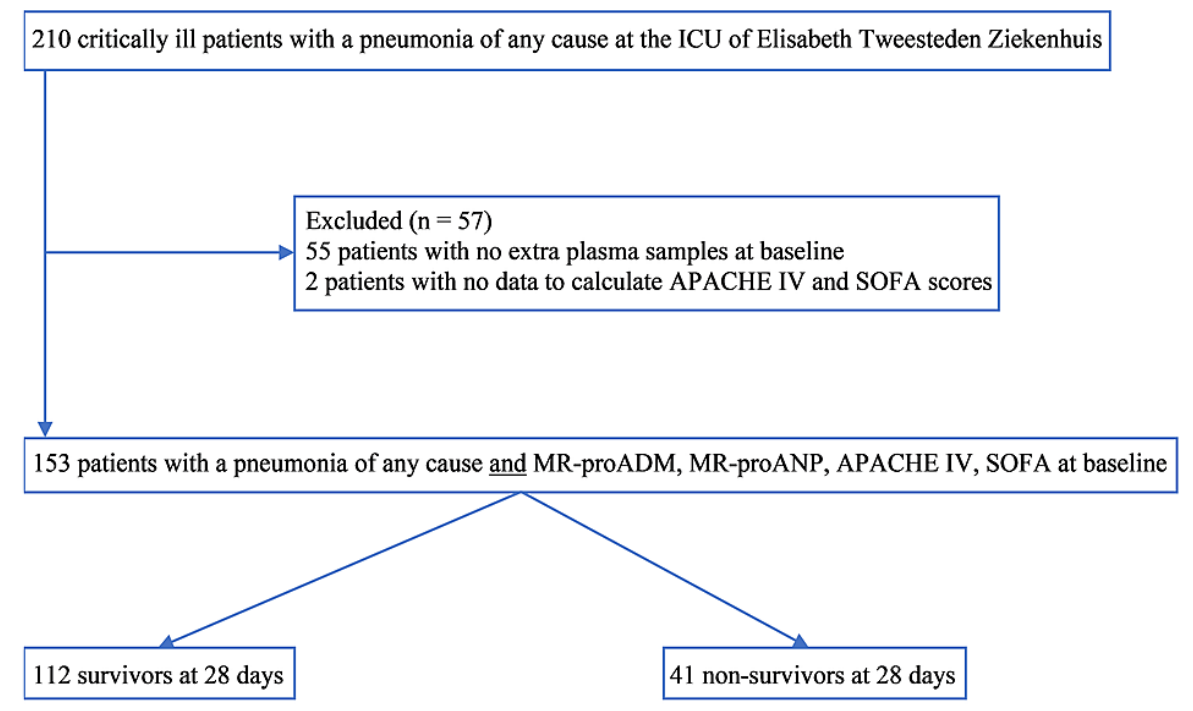

FIGURE 1: Patient flow diagram.

ICU: intensive care unit; APACHE IV: Acute Physiology and Chronic Health Evaluation IV; SOFA: Sequential Organ Failure Assessment; MR-proADM: mid-regional proadrenomedullin; MR-proANP: mid-regional proatrial natriuretic peptide

Demographics and clinical characteristics of these 153 patients, including MR-proADM, MR-proANP, APACHE IV, and SOFA score at baseline are shown in Table 1. The 28-day all-cause mortality was 26.8\%. Patients were divided into survivors and nonsurvivors with regards to survival for up to 28 days. Both groups were comparable except for older age and higher APACHE IV in nonsurvivors. There was no significant difference in SOFA score on the first day between both groups. Bacterial pathogens could be detected in 64 patients (41.8\%). Gram-positive microorganisms were found in 18 patients (Streptococcus pneumoniae $\mathrm{n}=14$, Enterococcus spp. $\mathrm{n}=2$, others $\mathrm{n}=2$ ). Gram-negative microorganisms were found in 46 patients (Haemophilus influenzae $\mathrm{n}=8$, Escherichia coli $\mathrm{n}=8$, Klebsiella spp. $\mathrm{n}=8$, Pseudomonas aeruginosa $\mathrm{n}=$ 


\section{Cureus}

6, Enterobacter spp. $\mathrm{n}=4$, Haemophilus parainfluenza $\mathrm{n}=2$, Moraxella catarrhalis $\mathrm{n}=2$, others $\mathrm{n}=8$ ). The database revealed no data on viral or fungal infections.

\begin{tabular}{|c|c|c|c|c|}
\hline & Total & Survivors & Nonsurvivors & P-value \\
\hline & $(\mathrm{N}=153)$ & $(\mathrm{N}=112)$ & $(\mathrm{N}=41)$ & \\
\hline Age (years) (median, IQR) & $65(53-74)$ & $63(51-71)$ & 69 (63-78) & 0.003 \\
\hline Male gender (N, \%) & $94(61.4 \%)$ & $66(58.9 \%)$ & $28(68.3 \%)$ & 0.292 \\
\hline \multicolumn{5}{|l|}{ Patient category (N, \%) } \\
\hline Medical & $103(67.3 \%)$ & $71(63.4 \%)$ & $32(78 \%)$ & 0.090 \\
\hline Surgical & 27 (17.6\%) & $20(17.9 \%)$ & $7(17.1 \%)$ & \\
\hline Trauma & $23(15 \%)$ & $21(18.8 \%)$ & $2(4.9 \%)$ & \\
\hline \multicolumn{5}{|l|}{ Preexisting comorbidities (N, \%) } \\
\hline Congestive heart failure & $15(9.8 \%)$ & $8(7.1 \%)$ & $7(17.1 \%)$ & 0.067 \\
\hline COPD & $40(26.1 \%)$ & $25(22.3 \%)$ & $15(36.6 \%)$ & 0.075 \\
\hline Diabetes mellitus & $26(17 \%)$ & $17(15.2 \%)$ & $9(22 \%)$ & 0.323 \\
\hline Cerebrovascular disease & 37 (24.2\%) & $23(20.5 \%)$ & $14(34.1 \%)$ & 0.082 \\
\hline Malignancy & $21(13.7 \%)$ & $14(12.5 \%)$ & 7 (17.1\%) & 0.467 \\
\hline Chronic renal disease & $9(5.9 \%)$ & $5(4.5 \%)$ & $4(9.8 \%)$ & 0.218 \\
\hline \multicolumn{5}{|l|}{ Type of pneumonia (N, \%) } \\
\hline Community-acquired pneumonia & $60(39.2 \%)$ & $43(38.4 \%)$ & $17(41.5 \%)$ & 0.917 \\
\hline Hospital-acquired pneumonia & $76(49.7 \%)$ & $56(50 \%)$ & $20(48.8 \%)$ & \\
\hline Ventilator-associated pneumonia & $17(11.1 \%)$ & $13(11.6 \%)$ & $4(9.8 \%)$ & \\
\hline \multicolumn{5}{|l|}{ Microbiology } \\
\hline Gram-positive (N, \%) & $18(11.8 \%)$ & $14(12.5 \%)$ & $4(9.8 \%)$ & 0.151 \\
\hline Gram-negative (N, \%) & 46 (30\%) & 38 (33.9\%) & $8(19.5 \%)$ & \\
\hline No bacterial pathogens (N, \%) & 89 (58.2\%) & $60(53.6 \%)$ & $29(70.7 \%)$ & \\
\hline \multicolumn{5}{|l|}{ Severity of illness } \\
\hline Sepsis-3, sepsis (N, \%) & 148 (96.7\%) & 109 (97.3\%) & 39 (95.1\%) & 0.498 \\
\hline Sepsis-3, septic shock (N, \%) & $27(17.8 \%)$ & $18(16.1 \%)$ & 9 (22.5\%) & 0.361 \\
\hline APACHE IV (points) (median, IQR) & $73(53-86)$ & $65(50-84)$ & $81(61-100)$ & 0.003 \\
\hline SOFA score (points) (median, IQR) & $5(3-8)$ & $5(3-7)$ & $6(3-10)$ & 0.441 \\
\hline \multicolumn{5}{|l|}{ Treatment upon diagnosis (N, \%) } \\
\hline Mechanical ventilation & 125 (81.7\%) & 90 (80.4\%) & 35 (85.4\%) & 0.478 \\
\hline Vasopressor use & $144(94.1 \%)$ & $105(93,8 \%)$ & 39 (95.1\%) & 0.749 \\
\hline Renal replacement therapy & $7(4.6 \%)$ & $5(4.5 \%)$ & $2(4.9 \%)$ & 0.914 \\
\hline \multicolumn{5}{|l|}{ Length of stay } \\
\hline ICU LOS (days) (median, IQR) & $9(5-18)$ & $9(4-22)$ & $9(5-15)$ & 0.754 \\
\hline \multicolumn{5}{|l|}{ Biomarkers } \\
\hline WBC, $10^{9} / \mathrm{L}$, (median, IQR) & $12.6(9.3-17.6)$ & $12.3(9.3-16.5)$ & $13.5(9.5-19)$ & 0.407 \\
\hline CRP (mg/L), (median, IQR) & $142(81.8-251)$ & $158(82-267)$ & $111(66-230)$ & 0.161 \\
\hline
\end{tabular}




\section{Cureus}

\begin{abstract}
PCT (ng/mL), (median, IQR)
Lactate (mmol/L), (median, IQR)

MR-proADM (nmol/L), (median, IQR)
\end{abstract}

MR-proANP (pmol/L), (median, IQR)

$0.7(0.2-4.3)$
$1.3(1-1.8)$
$1.3(0.9-2.5)$
$159.8(87.7-313)$

$159.8(87.7-313)$
$0.6(0.2-3.1)$

$1.3(1-1.8)$

$1.3(0.9-2.1)$

$141.7(79.1-208)$
$1.3(0.3-12.2)$

0.066

0.147

0.017

$1.8(1-3.3)$

0.001

\section{TABLE 1: Clinical characteristics of patients at baseline with regards to survival up to 28 days.}

All continuous data are presented as median (interquartile range) and categorical data as number (percentage).

COPD: chronic obstructive pulmonary disease; APACHE IV: Acute Physiology and Chronic Health Evaluation IV; SOFA: Sequential Organ Failure Assessment; ICU LOS: length of stay at the intensive care; WBC: white blood cell; CRP: C-reactive protein; PCT: procalcitonin; MR-proADM: midregional proadrenomedullin; MR-proANP: mid-regional proatrial natriuretic peptide

\section{Association between biomarkers at baseline and 28-day mortality}

Nonsurvivors at 28 days had significantly higher concentrations of MR-proADM and MR-proANP at baseline than survivors (Table 1). WBC, CRP, PCT, and lactate concentrations were not significantly different. The area under the curve (AUC) in ROC analysis for the prediction of 28-day mortality of baseline MR-proADM $(0.63 ; 95 \%$ confidence interval $(\mathrm{CI}): 0.53-0.73 ; \mathrm{p}=0.017)$ ) and MR-proANP $(0.68 ; 95 \% \mathrm{CI}: 0.59-0.78 ; \mathrm{p}=$ $0.001)$ ) was low (Table 2). AUCs for APACHE IV and SOFA were 0.66 (95\% CI: $0.56-0.76 ; p=0.003$ ) and 0.54 (95\% CI: 0.43-0.65; $\mathrm{p}=0.444$ ), respectively (Figure 2) (Table 2). Combinations of biomarkers and scores did not yield a higher AUC (Table 2). Due to a low AUC in the ROC analysis, no optimal cut-off points could be calculated. MR-proADM, MR-proANP, APACHE IV, and SOFA were divided into tertiles. The highest tertile of MR-proADM, MR-proANP, and APACHE IV on day 1 had the strongest association in predicting 28-day mortality compared to patients with the two lower tertiles in univariable Cox regression analysis (Table 3). SOFA score was not a significant predictor of 28-day mortality in univariable analysis and was excluded from the multivariable analysis. The highest tertiles baseline MR-proADM and MR-proANP were not significant predictors for 28-day mortality in a multivariable model with age and APACHE IV. Only APACHE IV had a significant contribution (hazard ratio [HR]: 2.07, 95\% CI: 1.11-3.86; $\mathrm{p}=0.021$ ) (Table 3). There were no signs of high correlations between the predictor variables in the model. Tolerance values were between 0.75 and 0.98 (Table 4$)$.

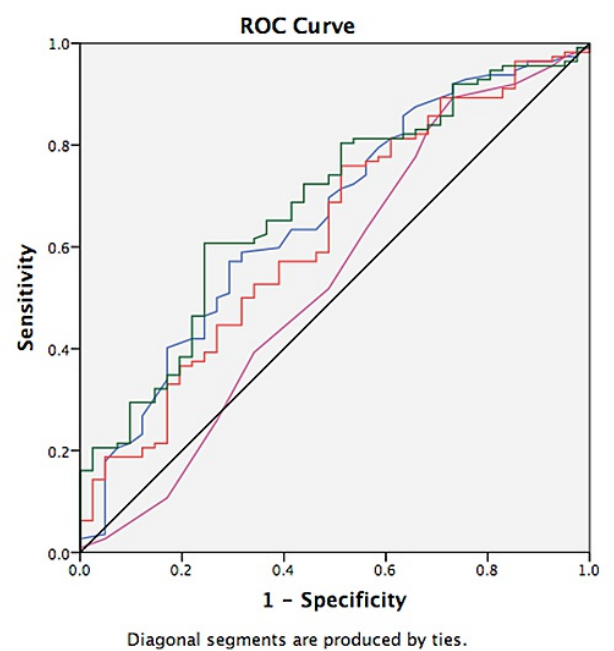

FIGURE 2: Receiver operating characteristics curve for biomarkers/clinical scores on day one in predicting 28-day mortality.

Red line = MR-proADM (AUC: $0.63 ; 95 \% \mathrm{Cl}$ : 0.53-0.73; $p=0.017$ ), green line = MR-proANP (AUC: $0.68 ; 95 \%$ Cl: $0.59-0.78 ; p=0.001$ ), blue line = APACHE IV (AUC: $0.66 ; 95 \% \mathrm{Cl}:-0.56-0.76 ; p=0.003$ ), violet line $=$ SOFA (AUC: $0.54 ; 95 \% \mathrm{Cl}: 0.43-0.65 ; p=0.444$ ), black line = reference line.

MR-proADM: mid-regional proadrenomedullin; MR-proANP: mid-regional proatrial natriuretic peptide; APACHE IV: Acute Physiology and Chronic Health Evaluation IV; SOFA: Sequential Organ Failure Assessment 


\section{Cureus}

\begin{tabular}{|c|c|c|c|c|}
\hline Biomarker or clinical score & Patients (N) & Mortality (N) & AUC (95\% Cl) & P-value \\
\hline MR-proADM & 153 & 41 & $0.63(0.53-0.73)$ & 0.017 \\
\hline MR-proANP & 153 & 41 & $0.68(0.59-0.78)$ & 0.001 \\
\hline APACHE IV & 153 & 41 & $0.66(0.56-0.76)$ & 0.003 \\
\hline SOFA & 153 & 41 & $0.54(0.43-0.65)$ & 0.444 \\
\hline APACHE IV + MR-proADM & 153 & 41 & $0.68(0.58-0.78)$ & 0.001 \\
\hline APACHE IV + MR-proANP & 153 & 41 & $0.69((0.59-0.79)$ & $<0.001$ \\
\hline SOFA + MR-proADM & 153 & 41 & $0.61(0.50-0.72)$ & 0.039 \\
\hline SOFA + MR-proANP & 153 & 41 & $0.68(0.59-0.78)$ & 0.001 \\
\hline MR-proADM + MR-proANP & 153 & 41 & $0.68(0.59-0.77)$ & 0.001 \\
\hline
\end{tabular}

\section{TABLE 2: Prediction of 28-day mortality by biomarkers and clinical scores on the first day.}

Association between biomarkers and clinical scores with 28-day mortality by receiver operating characteristics curves.

AUC: area under the curve; $95 \% \mathrm{Cl}$ : 95\% confidence interval; MR-proADM: mid-regional proadrenomedullin; MR-proANP: mid-regional proartrial natriuretic peptide; APACHE IV: Acute Physiological and Chronic Health Evaluation IV; SOFA: Sequential Organ Failure Assessment

\begin{tabular}{|c|c|c|c|c|}
\hline & \multicolumn{2}{|c|}{ Univariable analysis } & \multicolumn{2}{|c|}{ Muitivariable analysis } \\
\hline & HR $(95 \%$ CI) & P-value & HR $(95 \%$ CI) & P-value \\
\hline Age & $1.03(1.01-1.06)$ & 0.014 & $1.02(0.99-1.05)$ & $0.15 /$ \\
\hline \multicolumn{5}{|l|}{ SOFA } \\
\hline Low $\left(1^{\text {st }}+2^{\text {nd }}\right.$ tertiles $)$ & 1.0 (Reference) & & - & - \\
\hline High (3 $3^{\text {rd }}$ tertile) & $1.25(0.68-2.32)$ & 0.476 & - & - \\
\hline \multicolumn{5}{|l|}{ APACHE IV } \\
\hline Low $\left(1^{\mathrm{st}}+2^{\text {na }}\right.$ tertiles $)$ & 1.0 (Reterence) & & 1.0 (Reterence) & \\
\hline High ( $3^{\text {rd }}$ tertile) & $2.22(1.20-4.10)$ & 0.011 & $2.07(1.11-3.86)$ & 0.021 \\
\hline \multicolumn{5}{|l|}{ MR-proADM day 1} \\
\hline Low ( $1^{\text {st }}+2^{\text {nd }}$ tertiles $)$ & 1.0 (Reference) & & 1.0 (Reference) & \\
\hline High (3 $3^{\text {rd }}$ tertile) & $2.12(1.15-3.91)$ & 0.017 & $1.09(0.50-2.38)$ & 0.823 \\
\hline \multicolumn{5}{|l|}{ MR-proANP day 1} \\
\hline Low $\left(1^{\text {st }}+2^{\text {nd }}\right.$ tertiles $)$ & 1.0 (Reference) & & 1.0 (Reference) & \\
\hline High (3 $3^{\text {rd }}$ tertile) & $2.42(1.31-4.47)$ & 0.005 & $1.87(0.91-3.83)$ & 0.089 \\
\hline
\end{tabular}

TABLE 3: Univariable and multivariable Cox regression models for the prediction of 28-day mortality with baseline biomarker values.

HR: hazard ratio; Cl: confidence interval; SOFA: Sequential Organ Failure Assessment; APACHE IV: Acute Physiology and Chronic Health Evaluation IV; MR-proADM: mid-regional proadrenomedullin; MR-proANP: mid-regional proatrial natriuretic peptide 


\section{Cureus}

Tolerance value

Age

APACHE IV

MR-proADM

MR-proANP
0.79

0.98

0.69

0.75

TABLE 4: Collinearity statistics of biomarkers/clinical scores on the first day in multivariable Cox regression model.

\section{Association between biomarker kinetics and 28-day mortality}

Clearance percentages in five days of MR-proADM and MR-proANP were calculated and compared between survivors and nonsurvivors in 28 days. Clearances of MR-proADM and MR-proANP were significantly higher in survivors compared to nonsurvivors in 28 days (Table 5). MR-proADM and MR-proANP clearance had low accuracy to identify nonsurvivors in ROC curves with AUC of 0.66 (95\% CI: $0.56-0.76 ; p=0.004$ ) and 0.68 (95\% CI: 0.257-0.78; $=0.002$ ), respectively (Figure 3) (Table 6). A combination of biomarker clearance did not yield a substantially higher AUC (Table 6). Clearances of MR-proADM and MR-proANP were divided into tertiles. Univariable Cox regression analysis demonstrated that the lowest MR-proADM and MR-proANP clearance (first tertile) in five days had a strong association in predicting 28-day mortality compared to patients with the combined second and third tertiles (Table 7). SOFA score was excluded from further multivariable analysis. Low clearances of MR-proADM and MR-proANP (first tertiles) were significant predictors for 28-day mortality (HR: 2.38 ; 95\% CI: $1.21-4.70$; $\mathrm{p}=0.013$ and HR: 2.27 ; 95\% CI: $1.16-4.46$; $\mathrm{p}=$ 0.017 , respectively) in a model with age and APACHE IV (Table 7). There were no signs of high correlations between the predictor variables, and tolerance values were between 0.94 and 0.98 (Table 8 ).

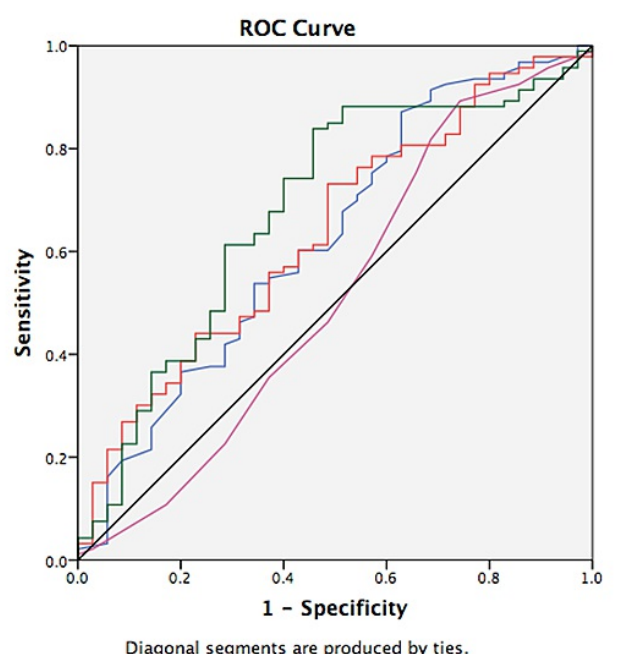

FIGURE 3: Receiver operating characteristics curve biomarker clearance in five days in predicting 28-day mortality.

Red line = MR-proADM clearance (AUC: $0.66 ; 95 \% \mathrm{Cl}: 0.56-0.76 ; p=0.004)$, green line $=$ MR-proANP clearance (AUC: $0.68 ; 95 \% \mathrm{Cl}$ : 0.57-0.79; $\mathrm{p}=0.001$ ), blue line = APACHE IV (AUC: $0.66 ; 95 \% \mathrm{Cl}$ : $-0.56-0.76 ; \mathrm{p}$ $=0.003$ ), violet line $=$ SOFA (AUC: $0.54,95 \% \mathrm{Cl}: 0.43-0.65 ; p=0.444$ ), black line $=$ reference line

MR-proADM: mid-regional proadrenomedullin; MR-proANP: mid-regional proatrial natriuretic peptide; APACHE IV: Acute Physiology and Chronic Health Evaluation IV; SOFA: Sequential Organ Failure Assessment 


\section{Cureus}

\begin{tabular}{|c|c|c|c|}
\hline \multirow{2}{*}{ 28-day survival } & Survivors & Nonsurvivors & P-value \\
\hline & $N=112$ & $N=41$ & \\
\hline MR-proADM clearance (\%) & $34 \%(16-50)$ & $16 \%(-6-37)$ & 0.004 \\
\hline MR-proANP clearance (\%) & $14 \%(0-34)$ & $-9 \%(-28-18)$ & 0.002 \\
\hline
\end{tabular}

\section{TABLE 5: MR-proADM and MR-proANP clearance in five days.}

All continuous data are presented as median percentage (interquartile range)

MR-proADM: mid-regional proadrenomedullin; MR-proANP: mid-regional proatrial natriuretic peptide

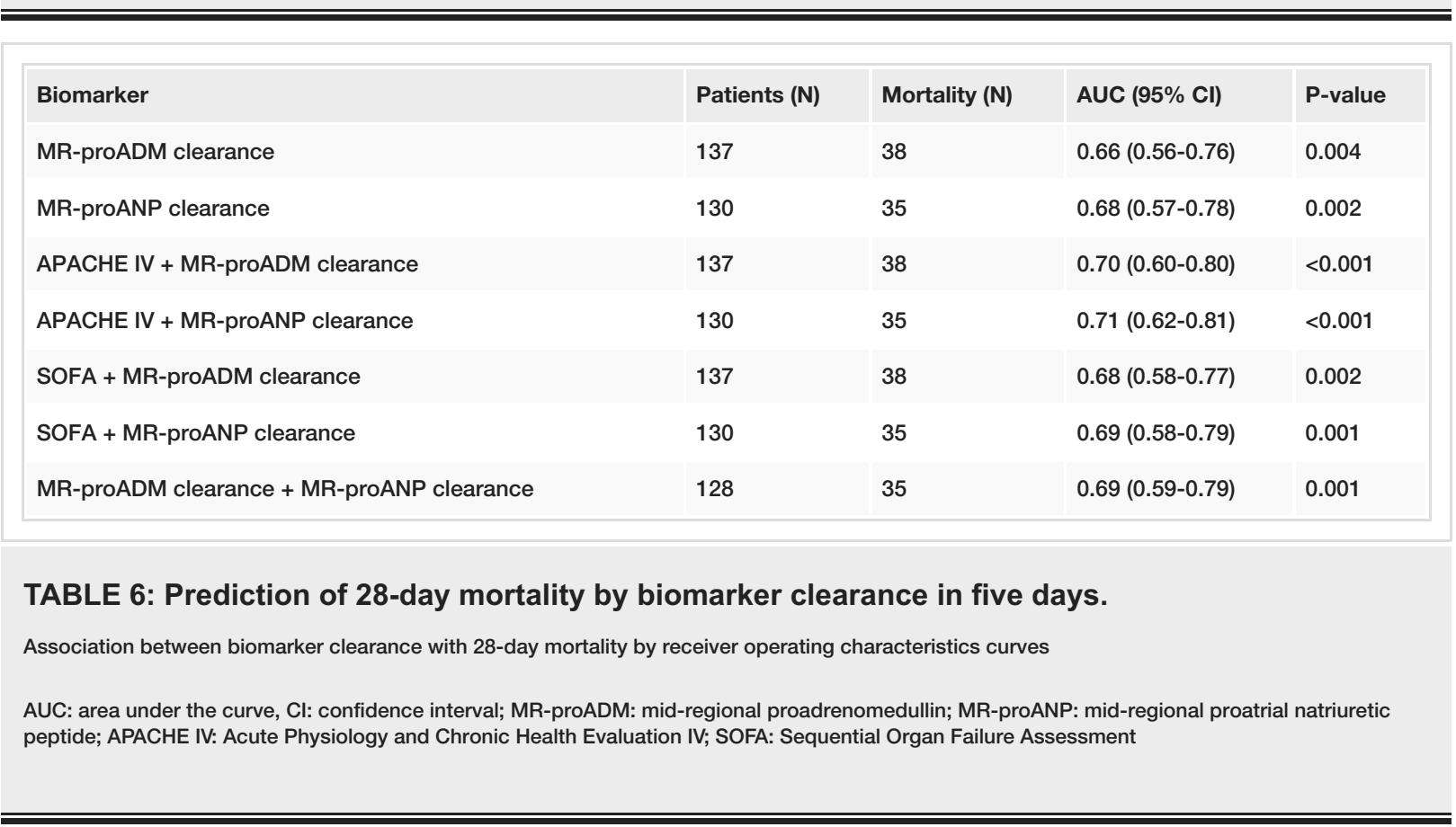




\section{Cureus}

\begin{tabular}{|c|c|c|c|c|}
\hline & \multicolumn{2}{|c|}{ Univariable analysis } & \multicolumn{2}{|c|}{ Multivariable analysis } \\
\hline & HR (95\% Cl) & P-value & HR (95\% Cl) & P-value \\
\hline Age & $1.03(1.01-1.06)$ & 0.014 & $1.03(1.01-1.06)$ & 0.033 \\
\hline \multicolumn{5}{|l|}{ SOFA } \\
\hline Low $\left(1^{\text {st }}+2^{\text {nd }}\right.$ tertiles $)$ & 1.0 (Reference) & & - & - \\
\hline High ( $3^{\text {rd }}$ tertile) & $1.25(0.68-2.32)$ & 0.476 & - & - \\
\hline \multicolumn{5}{|l|}{ APACHE IV } \\
\hline Low $\left(1^{\text {st }}+2^{\text {nd }}\right.$ tertiles $)$ & 1.0 (Reference) & & 1.0 (Reference) & \\
\hline High ( $3^{\text {rd }}$ tertile) & $2.22(1.20-4.10)$ & 0.011 & $1.71(0.87-3.37)$ & 0.122 \\
\hline \multicolumn{5}{|l|}{ MR-proADM clearance } \\
\hline $2^{\text {nd }}+3^{\text {rd }}$ tertiles ( $\geq 17 \%$ drop) & 1.0 (Reference) & & 1.0 (Reference) & \\
\hline $1^{\text {st }}$ tertile (<17\% drop) & $2.52(1.33-4.76)$ & 0.004 & $2.38(1.21-4.70)$ & 0.013 \\
\hline \multicolumn{5}{|l|}{ MR-proANP clearance } \\
\hline $2^{\text {nd }}+3^{\text {rd }}$ tertiles ( $\geq 0 \%$ drop $)$ & 1.0 (Reference) & & 1.0 (Reference) & \\
\hline $1^{\text {st }}$ tertile $(<0 \%$ drop $)$ & $2.80(1.44-5.45)$ & 0.002 & $2.27(1.16-4.46)$ & 0.017 \\
\hline
\end{tabular}

TABLE 7: Univariable and multivariable Cox regression models for the prediction of 28-day mortality with biomarker clearance in five days.

HR: hazard ratio; Cl: confidence interval; SOFA: Sequential Organ Failure Assessment; APACHE IV: Acute Physiology and Chronic Health Evaluation IV; MR-proADM: mid-regional proadrenomedullin; MR-proANP: mid-regional proatrial natriuretic peptide

\begin{tabular}{|l|l|}
\hline \hline & Tolerance value \\
\hline Age & 0.94 \\
\hline APACHE IV & 0.94 \\
MR-proADM clearance & 0.98 \\
\hline MR-proANP clearance & 0.98 \\
\hline
\end{tabular}

\section{TABLE 8: Collinearity statistics of biomarker clearance in five days in multivariable Cox} regression model.

APACHE IV: Acute Physiology and Chronic Health Evaluation IV; MR-proADM: mid-regional proadrenomedullin; MR-proANP: mid-regional proatrial natriuretic peptide

\section{Discussion}

The primary aim of our study was to investigate the prognostic value of baseline MR-proADM and MRproANP compared with APACHE IV and SOFA scores to predict 28-day mortality in a well-described cohort of critically ill patients with pneumonia. The secondary aim was the prediction of 28-day mortality by clearances of MR-proADM and MR-proANP in five days compared with both severity scores. We reported two main findings. First, single baseline MR-proADM and MR-proANP levels were not superior to APACHE IV in both ROC curves and in a multivariable Cox regression model for the prediction of 28-day mortality. SOFA score performed poorly in identifying nonsurvivors. Second, MR-proADM and MR-proANP clearance in five days performed better in predicting 28-day mortality in a model with age and APACHE IV. However, APACHE IV and SOFA scores require time and effort to collect data and are only available after 24 hours from admission [6]. A single baseline biomarker, with the ability to provide prognostic information early after 
admission, would have an advantage. Unfortunately, single baseline MR-proADM and MR-proANP did not perform well in our study. The addition of a single baseline MR-proADM and MR-proANP to the APACHE IV and SOFA models did not increase their predictive value. Clearances of biomarkers MR-proADM and MRproANP performed better in the Cox regression model but required five days to calculate in our study.

Our findings of a limited value of baseline MR-proADM and MR-proANP in predicting short-term survival in critically ill patients with pneumonia are in contrast with several studies. These two biomarkers have been studied frequently in patients with respiratory infections $[7,9,13,16,17]$. However, most patients were not admitted to the ICU $[7,9,13,16]$. Baseline MR-proADM and MR-proANP were studied in an observational cohort study of 728 CAP patients admitted to the emergency department (ED) [9]. Only 18 patients were admitted to the ICU. MR-proADM had the best performance for the prediction of 28-day mortality (HR: 3.67 and AUC: 0.85 ). MR-proADM proved to have the highest HR in predicting 28-day mortality in an observational cohort study of 1,175 ED patients [21]. Only 32 patients were admitted to the ICU. Baseline MR-proADM performed well in comparison with APACHE II and Simplified Acute Physiology Score II (SAPS II) in another observational study [22]. Pneumonia patients were not selected in this study as all patients admitted to the ICU were included. Our findings of better performance of MR-proADM clearance in five days as a prognostic factor in predicting mortality were supported by a study in which clearance of MR-proADM of $30 \%$ or more in five days had a higher survival probability in 100 days among septic ICU patients [14].

MR-proADM and MR-proANP are predominantly cardiovascular biomarkers. A possible reason for the elevation of cardiovascular biomarkers in acute pulmonary disease may be transient pulmonary hypertension, resulting in right heart strain [7,9]. An explanation for the observed lower prognostic value of the biomarkers in our cohort could be underlying cardiac failure [23]. The database was searched for comorbidities, and only 15 patients had congestive heart failure as comorbidity, but there were no significant differences between both groups. Septic cardiomyopathy could be another reason for elevated biomarkers, and almost all of our patients were septic according to Sepsis-3 definitions, but well balanced between survivors and nonsurvivors. Renal failure is another reason for the elevation of MR-proADM and MR-proANP, most probably due to inappropriate renal clearance or increased strain on the atria due to fluid overload $[9,24]$. A small number of patients had chronic renal disease as comorbidity, and a small portion of the cohort was on renal replacement therapy the first day. As day one biomarkers had low prognostic accuracy regarding survival, biomarkers may need time to differentiate between survival and death. Indeed, MR-proADM and MR-proANP clearance in five days performed better in predicting mortality. Persistent high levels of biomarkers in nonsurvivors may be due to persistent synthesis and release due to active organ dysfunction [14]. This hypothesis is supported by a prospective cohort study with 114 septic critically ill patients with cancer [25]. MR-proADM levels decreased from baseline to follow-up (four to seven days later) in survivors and increased in nonsurviving patients in this study. Moreover, MR-proANP levels increased in five days in nonsurvivors, resulting in a negative calculated clearance percentage of MR-proANP in our cohort. Persistently increased levels of MR-proANP in 10 days following VAP onset were also reported in nonsurviving patients in another study [17].

Some limitations of our study need to be addressed. First, we performed a retrospective observational study in a cohort of critically ill patients with pneumonia selected from the SAPS database. We must rely on older data of clinical practice leading to potential observational bias. Second, by selecting 153 patients out of 210 potential eligible critically ill patients with pneumonia and index tests, we introduced selection bias. Third, our study population is relatively small and heterogeneous in source as patients with CAP, HAP, and VAP were included. These subgroups were too small to analyze by diagnostic subgroup. Both observational and selection bias may have led to potential underestimation of the prognostic performance of MR-proADM and MR-proANP, and we, therefore, plan to conduct a larger prospective observational study in the near future.

A strong feature of our analysis is that it is a real-life study performed in a mixed surgical and medical ICU with patients with pneumonia consisting of CAP, HAP, and VAP, reflecting routine clinical ICU practice.

\section{Conclusions}

Single baseline MR-proADM and MR-proANP were not superior to APACHE IV in predicting 28-day mortality in a mixed population of critically ill patients with pneumonia. SOFA score was not a significant predictor of 28-day mortality. The predictive value of serial-measured MR-proADM and MR-proANP in predicting 28-day mortality in a model with age and APACHE IV exceeded those of single baseline MRproADM and MR-proANP measurements in this population. Therefore, clearance of MR-proADM and MRproANP in time may be better used for prognostication instead of single values.

\section{Additional Information \\ Disclosures}

Human subjects: Consent was obtained or waived by all participants in this study. Ethics committee of the VU University Medical Centre (Amsterdam, Netherlands) issued approval NL-nummer: NL26543.029.09. Ethics approval and consent to participate: The SAPS study protocol was approved for all centers by the ethics committee of the VU University Medical Centre (Amsterdam, Netherlands) (NL-number: 
NL26543.029.09). All patients agreed by informed consent that daily blood samples were stored and could be used for further research. Animal subjects: All authors have confirmed that this study did not involve animal subjects or tissue. Conflicts of interest: In compliance with the ICMJE uniform disclosure form, all authors declare the following: Payment/services info: All authors have declared that no financial support was received from any organization for the submitted work. Financial relationships: All authors have declared that they have no financial relationships at present or within the previous three years with any organizations that might have an interest in the submitted work. Other relationships: All authors have declared that there are no other relationships or activities that could appear to have influenced the submitted work.

\section{References}

1. Wunderink RG, Waterer GW: Clinical practice. Community-acquired pneumonia. N Engl J Med. 2014, 370:543-51. 10.1056/NEJMcp1214869

2. Musher DM, Thorner AR: Community-acquired pneumonia. N Engl J Med. 2014, 371:1619-28. 10.1056/NEJMra1312885

3. Mandell LA, Wunderink RG, Anzueto A, et al.: Infectious Diseases Society of America/American Thoracic Society consensus guidelines on the management of community-acquired pneumonia in adults. Clin Infect Dis. 2007, 44 Suppl 2:S27-72. 10.1086/511159

4. Rodriguez A, Lisboa T, Blot S, Martin-Loeches I, Solé-Violan J, De Mendoza D, Rello J: Mortality in ICU patients with bacterial community-acquired pneumonia: when antibiotics are not enough. Intensive Care Med. 2009, 35:430-8. 10.1007/s00134-008-1363-6

5. American Thoracic Society, Infectious Diseases Society of America: Guidelines for the management of adults with hospital-acquired, ventilator-associated, and healthcare-associated pneumonia. Am J Respir Crit Care Med. 2005, 171:388-416. 10.1164/rccm.200405-644ST

6. Vincent JL, Moreno R: Clinical review: scoring systems in the critically ill . Crit Care. 2010, 14:207. $10.1186 /$ cc8204

7. Christ-Crain M, Morgenthaler NG, Stolz D, et al.: Pro-adrenomedullin to predict severity and outcome in community-acquired pneumonia [ISRCTN04176397]. Crit Care. 2006, 10:R96. 10.1186/cc4955

8. Suberviola B, Castellanos-Ortega A, Ruiz Ruiz A, Lopez-Hoyos M, Santibañez M: Hospital mortality prognostication in sepsis using the new biomarkers sUPAR and proADM in a single determination on ICU admission. Intensive Care Med. 2013, 39:1945-52. 10.1007/s00134-013-3056-Z

9. Krüger S, Ewig S, Giersdorf S, Hartmann O, Suttorp N, Welte T: Cardiovascular and inflammatory biomarkers to predict short- and long-term survival in community-acquired pneumonia: results from the German Competence Network, CAPNETZ. Am J Respir Crit Care Med. 2010, 182:1426-34. 10.1164/rccm.201003-04150C

10. Struck J, Tao C, Morgenthaler NG, Bergmann A: Identification of an adrenomedullin precursor fragment in plasma of sepsis patients. Peptides. 2004, 25:1369-72. 10.1016/j.peptides.2004.06.019

11. Morgenthaler NG, Struck J, Thomas B, Bergmann A: Immunoluminometric assay for the midregion of proatrial natriuretic peptide in human plasma. Clin Chem. 2004, 50:234-6. 10.1373/clinchem.2003.021204

12. Temmesfeld-Wollbrück B, Brell B, Dávid I, et al.: Adrenomedullin reduces vascular hyperpermeability and improves survival in rat septic shock. Intensive Care Med. 2007, 33:703-10. 10.1007/s00134-007-0561-y

13. Albrich WC, Dusemund F, Rüegger K, et al.: Enhancement of CURB65 score with proadrenomedullin (CURB65-A) for outcome prediction in lower respiratory tract infections: derivation of a clinical algorithm. BMC Infect Dis. 2011, 11:112. 10.1186/1471-2334-11-112

14. Valenzuela-Sánchez F, Valenzuela-Méndez B, Rodríguez-Gutiérrez JF, Estella-García Á, González-García MÁ: New role of biomarkers: mid-regional pro-adrenomedullin, the biomarker of organ failure . Ann Transl Med. 2016, 4:329. 10.21037/atm.2016.08.65

15. Morgenthaler NG, Struck J, Christ-Crain M, Bergmann A, Müller B: Pro-atrial natriuretic peptide is a prognostic marker in sepsis, similar to the APACHE II score: an observational study. Crit Care. 2005, 9:R3745. 10.1186/cc3015

16. Schuetz P, Wolbers M, Christ-Crain M, et al.: Prohormones for prediction of adverse medical outcome in community-acquired pneumonia and lower respiratory tract infections. Crit Care. 2010, 14:R106. 10.1186/cc9055

17. Boeck L, Eggimann P, Smyrnios N, et al.: Midregional pro-atrial natriuretic peptide and procalcitonin improve survival prediction in VAP. Eur Respir J. 2011, 37:595-603. 10.1183/09031936.00023810

18. de Jong E, van Oers JA, Beishuizen A, et al.: Efficacy and safety of procalcitonin guidance in reducing the duration of antibiotic treatment in critically ill patients: a randomised, controlled, open-label trial. Lancet Infect Dis. 2016, 16:819-27. 10.1016/S1473-3099(16)00053-0

19. Horan TC, Andrus M, Dudeck MA: CDC/NHSN surveillance definition of health care-associated infection and criteria for specific types of infections in the acute care setting. Am J Infect Control. 2008, 36:309-32. 10.1016/j.ajic.2008.03.002

20. von Elm E, Altman DG, Egger M, Pocock SJ, Gøtzsche PC, Vandenbroucke JP: The Strengthening the Reporting of Observational Studies in Epidemiology (STROBE) statement: guidelines for reporting observational studies. PLoS Med. 2007, 4:e296. 10.1371/journal.pmed.0040296

21. Saeed K, Wilson DC, Bloos F, et al.: Correction to: the early identification of disease progression in patients with suspected infection presenting to the emergency department: a multi-centre derivation and validation study. Crit Care. 2019, 23:255. 10.1186/s13054-019-2536-0

22. Bellia C, Agnello L, Lo Sasso B, Bivona G, Raineri MS, Giarratano A, Ciaccio M: Mid-regional proadrenomedullin predicts poor outcome in non-selected patients admitted to an intensive care unit. Clin Chem Lab Med. 2019, 57:549-55. 10.1515/cclm-2018-0645

23. Gegenhuber A, Struck J, Dieplinger B, et al.: Comparative evaluation of B-type natriuretic peptide, midregional pro-A-type natriuretic peptide, mid-regional pro-adrenomedullin, and Copeptin to predict 1-year 


\section{Cureus}

mortality in patients with acute destabilized heart failure. J Card Fail. 2007, 13:42-9.

10.1016/j.cardfail.2006.09.004

24. Vickery S, Price CP, John RI, Abbas NA, Webb MC, Kempson ME, Lamb EJ: B-type natriuretic peptide (BNP) and amino-terminal proBNP in patients with CKD: relationship to renal function and left ventricular hypertrophy. Am J Kidney Dis. 2005, 46:610-20. 10.1053/j.ajkd.2005.06.017

25. Debiane L, Hachem RY, Al Wohoush I, et al.: The utility of proadrenomedullin and procalcitonin in comparison to C-reactive protein as predictors of sepsis and bloodstream infections in critically ill patients with cancer. Crit Care Med. 2014, 42:2500-7. 10.1097/CCM.0000000000000526 